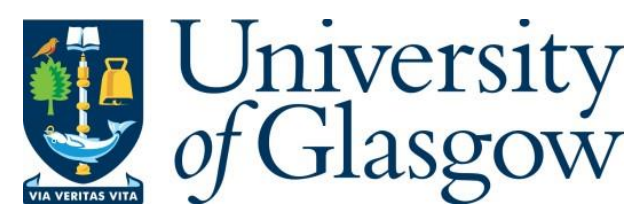

Konda, P. C. , Aidukas, T., Taylor, J. and Harvey, A. R. (2017) Miniature Fourier Ptychography Microscope using Raspberry Pi Camera and Hardware. Imaging and Applied Optics 2017, San Francisco, CA, USA, 26-29 Jun 2017.

There may be differences between this version and the published version. You are advised to consult the publisher's version if you wish to cite from it.

http://eprints.gla.ac.uk/145088/

Deposited on: 1 August 2017

Enlighten - Research publications by members of the University of Glasgow http://eprints.gla.ac.uk 


\title{
Miniature Fourier ptychography microscope using Raspberry Pi camera and hardware
}

\author{
Pavan Chandra Konda, Tomas Aidukas, Andrew R. Harvey* \\ Imaging Concepts Group, School of Physics and Astronomy, University of Glasgow, Scotland, G12 8QQ, UK \\ *Andy.Harvey@glasgow.ac.uk
}

\begin{abstract}
We report a Fourier ptychography setup using a raspberry pi camera detector and its lens in reversed configuration. In this work, data acquisition was performed by means of a raspberry pi 3 board which eliminates the requirement of a computer for data acquisition, thus allowing a miniaturized system for remote data acquisition to be built for around $£ 100$.

OCIS codes: (100.5070) Phase retrieval; (170.0180) Microscopy; (110.1758) Computational imaging
\end{abstract}

\section{Introduction}

Optical light microscopy is routinely used for disease diagnosis and progression monitoring. Conventional microscopes require expensive optical components to achieve the necessary image quality. In addition, they also suffer from numerous other limitations such as small field-of-view and shallow depth-of-field. Computational imaging techniques such as Fourier ptychography (FP) [1] and snapshot wavefront coding [2] were proposed to address these limitations. Here we present a novel implementation of FP which employs a low-cost raspberry pi camera module for data acquisition, thus reducing the cost and size of the system. Sophisticated reconstruction algorithms [3-5] were employed to compensate for the loss in information due to the Bayer pattern. The camera and the LED array were interfaced to the Raspberry Pi board which performs the data acquisition; thus, resulting in a compact standalone system. In the future, we intend to perform the data processing on the Raspberry Pi board, therefore the need for a computer is eliminated completely. Due to its cost and standalone capability, this device can be used in remote areas such as would be necessary in developing countries for the diagnosis of diseases such as malaria and cancer. This device can also be used inside incubator chambers in research labs due to its small size allowing researchers to perform longitudinal studies of events such as cell culture. We also intend to use these sensors in multi-aperture Fourier ptychography [6,7] systems in order to further decrease the cost of such systems.

In the next section we describe the Fourier ptychography principle and the reconstruction procedure adopted in this work. We then discuss our miniature FP configuration and present our system which was built using 3D printed parts and translation stages. We show some preliminary data recorded from our setup and validate our idea by means of numerical simulations of our experimental setup in Matlab. We are currently in the process of calibrating our experimental setup and analysing experimental data.

\section{FP principle and Miniature FP setup}

FP is a technique to achieve space-bandwidth product in gigapixels by performing time-sequential simulation of high-NA illumination on a low-NA system (Fig. 1c). This system offers large depth-of-field (DoF), longer working distances, aberration estimation and correction as well as estimation of the phase of the sample itself. A GerchbergSaxton-Fienup [8] type of algorithm [4] is used to stitch the low-NA images into a high-resolution complex field image. These algorithms do not consider sparsely sampled data such as the Bayer patterned image. Another algorithm for sparsely sampled data was proposed in [3], however, this was never implemented on a Bayer patterned image. We use a combination of the above two algorithms to achieve the best performance out of our system.

Our experimental setup employs the Raspberry Pi camera lens as an imaging objective in a finite conjugate mode. The distance between the lens and the sensor was fixed by placing them in a 3D printed holder (Fig. 1B). This holder sits above the sample stage on a 3D printed translation stage and an LED array sits underneath the sample stage. Since the field-of-view is large, an XY translation stage is not required, however this can be embedded into the system if required.

An adafruit LED array with $4 \mathrm{~mm}$ separation between the LEDs was used for illumination. The LED array and camera were interfaced using a raspberry pi 3 board. The distance between the LED array and the sample was chosen to be $60 \mathrm{~mm}$ to provide $69 \%$ overlap in frequency space. The focal length of the lens was measured as $4.3 \mathrm{~mm}$ 
and an aperture diameter of $2 \mathrm{~mm}$ was used. A magnification of 1.5 was chosen in order to achieve Nyquist sampling. The required system NA can therefore calculated to be 0.138 . The pixel size of the sensor is 1.2 microns but 2.4 microns was considered for sampling requirement due to the Bayer pattern. A low-NA raw image obtained with $10 \mathrm{~ms}$ exposure from this system using a red LED is shown in Fig.1D to demonstrate the Bayer pattern sampling.

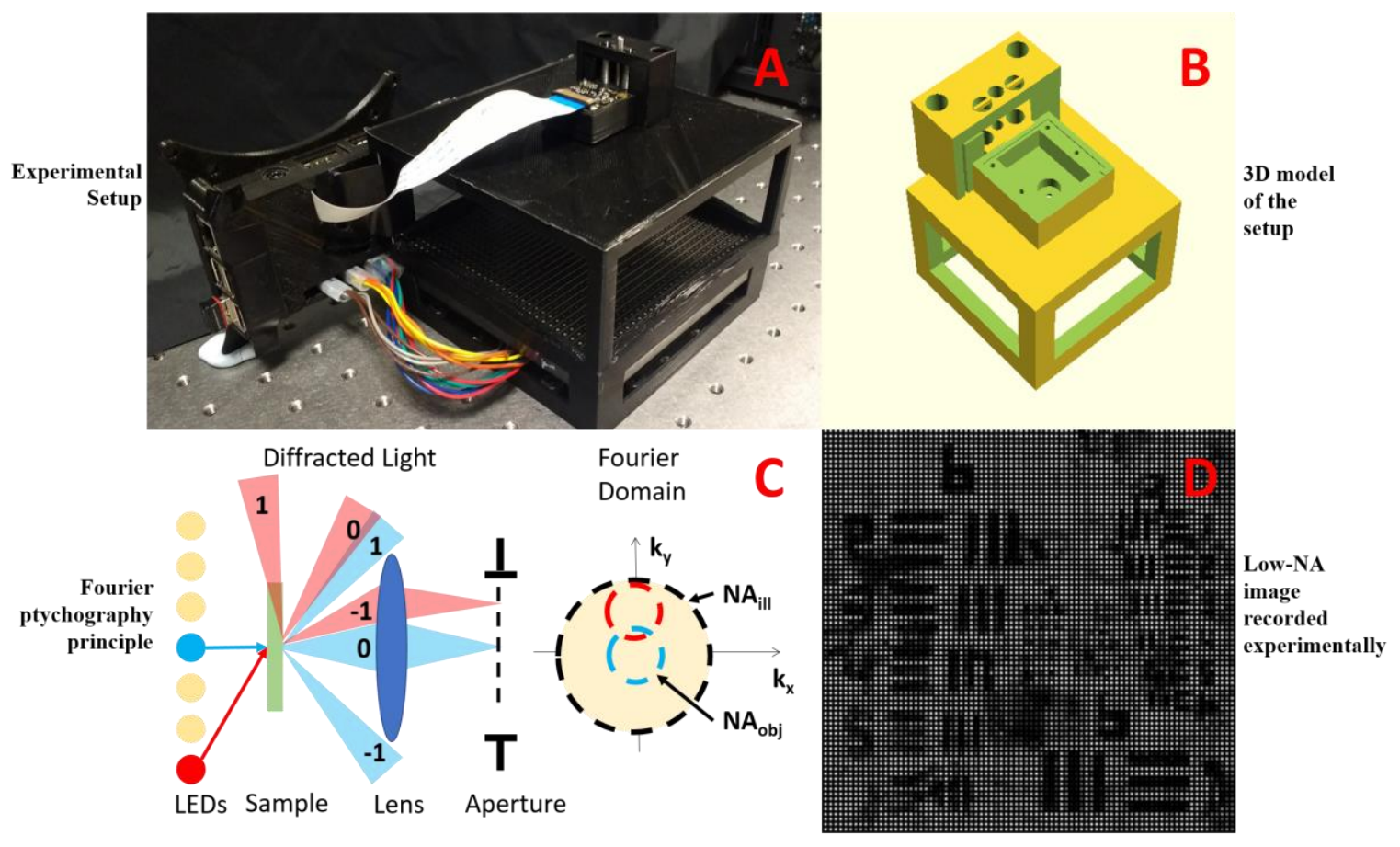

Figure 1 Fourier ptychography principle

\section{Simulation results}

We simulated our experimental setup with the parameters mentioned above. The noise of the detector at the exposure used to record the images was measured and found to have a standard deviation of 10 counts over 10 bit data. We impose similar noise in the simulated images with a Gaussian distribution with standard deviation of 5 times the amplitude value. A $21 \times 21$ LED array was chosen in the simulation in order to achieve a factor of 5 improvement in the NA. A second order Gauss-Newton reconstruction algorithm with a varying step size and sparse sampling with Bayer pattern was used. The results are shown in Fig. 2.

A synthetic scene with different amplitude and phase patterns was chosen as the sample. This sample was filtered according to the LED illumination angle and the pupil function to obtain the low-NA images. These were then stitched in the Fourier space using the reconstruction algorithm. The expected ideal reconstruction (sample with same set of frequency information as FP images) is shown in the bottom row of Fig. 2. The low-resolution image with Bayer pattern is shown in the first row. It can be seen that the red and blue channels are darker than the green due to the smaller number of pixels present in those channels. The low-resolution image without Bayer pattern (conventional) is shown in the fourth column and first row.

The amplitude and phase reconstructions of the scene in those individual channels are shown in the second and the third row respectively. It can be observed that the reconstruction quality in these different channels is high and comparable to the data without sparse sampling. The RMS error between the ideal image and the reconstructed image was calculated and is displayed on top of the images. This demonstrates that sparse sampling has negligible impact on the reconstruction. This is also due to the fact that we Nyquist sample the data by considering the Bayer pattern pixel instead of actual pixel size. This reduces the constraint of sparsely sampling and demonstrates that despite sparsely sampling the reconstruction, the quality can be unaffected. 


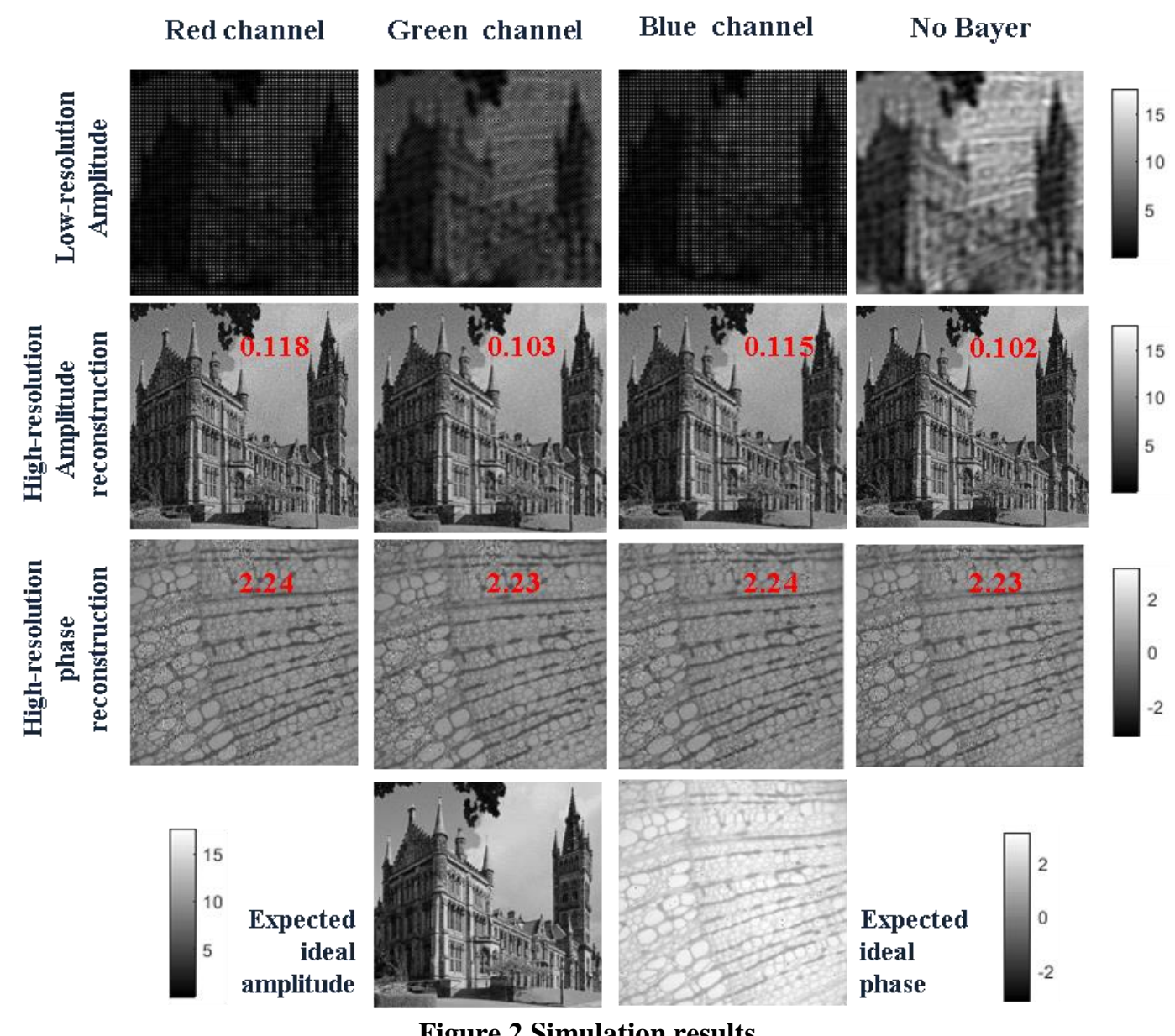

\section{Conclusion}

In this work we have demonstrated that a low-cost Raspberry Pi sensor can be used for FP microscopy with the help of simulations, despite having higher detector read noise and Bayer pattern sensor compared to sCMOS sensors. Using a Bayer pattern sensor also allows us to record all three colours in parallel. We demonstrated a 3D printed setup for the miniature FP microscope, though a large LED array was used in this experiment, it will be replaced by an array significantly smaller in future to keep the size to a minimum. Currently, only the data acquisition is performed by the raspberry-pi board; however, in the future, the data processing will be performed on the board. This makes for a very versatile microscope which can be useful for digital pathology in remote areas of developing countries and for incubation chambers in research.

\section{References}

1. R. Horstmeyer, G. Zheng, and C. Yang, "Wide-field, high-resolution Fourier ptychographic microscopy.," Nat. Photonics 7, 739-745 (2013).

2. P. Zammit, A. Harvey, and G. Carles, "Extended depth-of-field imaging and ranging in a snapshot," Optica 1, 209 (2014).

3. S. Dong, Z. Bian, R. Shiradkar, and G. Zheng, "Sparsely sampled Fourier ptychography.," Opt. Express 22, 5455-64 (2014).

4. L.-H. Yeh, L. Tian, Z. Liu, M. Chen, J. Zhong, and L. Waller, "Experimental robustness of Fourier Ptychographic phase retrieval algorithms," Opt. Express 23, (2015).

5. P. C. Konda, J. M. Taylor, and A. R. Harvey, "High-resolution microscopy with low-resolution objectives: correcting phase aberrations in Fourier ptychography," in Proc. SPIE 9630, Optical Systems Design 2015: Computational Optics, 96300X (September 23, 2015); doi:10.1117/12.2191338. (n.d.).

6. P. Konda, J. Taylor, and A. R. Harvey, "Multi-aperture Fourier Ptychography imaging in the near field," in Imaging and Applied Optics 2015, OSA Technical Digest (Online) (Optical Society of America, 2015), Paper CM3E.5. (n.d.).

7. P. C. Konda, J. M. Taylor, and A. R. Harvey, "Scheimpflug multi-aperture Fourier ptychography : coherent computational microscope with gigapixels / s data acquisition rates using 3D printed components," in Proc. SPIE 10076, High-Speed Biomedical Imaging and Spectroscopy: Toward Big Data Instrumentation and Management II, 100760R (February 22, 2017) (n.d.).

8. J. R. Fienup, "Reconstruction of a complex-valued object from the modulus of its Fourier transform using a support constraint," J. Opt. Soc. Am. A 4, 118-123 (1986). 